\title{
Impacto psicológico frente al COVID-19 en los internos de enfermería de prácticas pre-profesionales
}

\author{
María José Mera Navarrete \\ ORCID: https://orcid.org/0000-0003-2239-8470 \\ maria.mera78@est.ucacue.edu.ec \\ Carrera de Enfermería; Maestría en Gestión del \\ Cuidado del Posgrados de la Universidad Católica de \\ Cuenca. \\ Cuenca, Ecuador \\ Pedro Carlos Martínez Suárez \\ ORCID: https://orcid.org/0000-0002-1441-3821 \\ pmartinezs@ucacue.edu.ec \\ Laboratorio de Psicometría, Psicología Comparada \\ y Etología del Centro de Investigación, Innovación \\ y Transferencia de Tecnología (CIITT); Facultad de \\ Psicología Clínica, Universidad Católica de Cuenca \\ Cuenca, Ecuador
}

\author{
Isabel Cristina Mesa Cano \\ ORCID: https://orcid.org/0000-0003-3263-6145 \\ imesac@ucacue.edu.ec \\ Carrera de Enfermería; Maestría en Gestión del \\ Cuidado del Posgrados de la Universidad Católica de \\ Cuenca. \\ Cuenca, Ecuador \\ Andrés Alexis Ramírez Coronel \\ ORCID: https://orcid.org/0000-0002-6996-0443 \\ andres.ramirez@ucacue.edu.ec \\ Laboratorio de Psicometría, Psicología Comparada \\ y Etología del Centro de Investigación, Innovación \\ y Transferencia de Tecnología (CIITT); Carrera de \\ Enfermería; Maestría en Gestión del Cuidado del \\ Posgrados de la Universidad Católica de Cuenca. \\ Cuenca, Ecuador
}

Recibido (14/04/21), Aceptado (10/05/21)

Resumen:Se estudió el impacto psicológico frene al COVID - 19 en los internos de enfermería de prácticas pre - profesionales en relación a las variables sociodemográficas y los niveles de ansiedad, depresión y miedo. Para el análisis se consideró a 150 internos prácticos de la carrera de enfermería, para la evaluación de este estudio se utilizaron las escalas de STAI, IDER, FCV19S y K10. Los resultados exponen que existe mayor nivel de ansiedad en los internos que declararon no tener equipos de bioseguridad necesarios. Los internos que rotan en el área hospitalaria presentan mayores niveles de ansiedad, miedo y malestar psicológico compara con los internos del área comunitaria. Sin embargo, los niveles de depresión fueron ligeramente mayores en los internos de área comunitaria. No hubo diferencia significativa de los estados emocionales entre mujeres y varones.

Palabras Clave: Ansiedad, depresión, miedo al COVID-19, impacto psicológico.

\section{Psychological impact on covid-19 in nursing interns in pre-professional practices}

Abstract: The psychological impact of COVID-19 in nursing interns from pre-professional practices was studied in relation to sociodemographic variables and levels of anxiety, depression and fear. For the analysis 150 practical interns from the nursing career were considered, for the evaluation of this study the STAI, IDER, FCV 19S and K10 scales were used. The results show that there is a higher level of anxiety in the inmates who declared that they do not have the necessary biosafety equipment. The inmates who rotate in the hospital area present higher levels of anxiety, fear and psychological discomfort compared to the inmates in the community area. However, levels of depression were slightly higher in community area inmates. There was no significant difference in emotional states between women and men

Keywords: Anxiety, depression, fear of COVID-19, psychological impact.

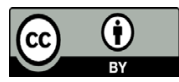




\section{Introducción}

A nivel mundial, la pandemia por el COVID - 19 ha tenido un impacto negativo en la vida de los seres humanos a nivel físico, social, emocional, y psicológico [1]. En el Ecuador [2] la aparición de la enfermedad no fue la excepción puesto que de acuerdo con lo reportado por Ministerio de Salud Pública (MSP) el primer caso de la enfermedad aprecio el 29 de febrero del 2020, importado por una viajera proveniente desde España [3]. Tras varios meses, el virus se ha extendido por todas las provincias originando colapso en el sistema de salud de varias ciudades y un impacto negativo en las dimensiones salud, economía, social, cultural y psicológico en toda la población ecuatoriana [4].

En este contexto, la medida de confinamiento, tomada por el gobierno para evitar la propagación del virus, conlleva a repercusiones en la salud mental de la población. No obstante, a nivel laboral ha provocado nuevas condiciones en donde los profesionales, incluyendo a los estudiantes del área de la salud, también se vieron afectados a nivel físico y emocional, debido a la vulnerabilidad en la que se encontraban y al temor que vivían ante lo desconocido de la situación actual [5]. De acuerdo con un estudio realizado la respuesta del personal que trabaja en primera línea frente al escenario actual, es de miedo, ansiedad y de estrés que desencadena un malestar psicológico y repercute en todos los aspectos de la vida [6].

La importancia del bienestar psicológico del ser humano reside en que es un elemento clave para el proceso de un excelente ajuste emocional, cognitivo y social, influyendo de manera considerable en todos los ámbitos de la vida diaria [7]. El bienestar psicológico es una práctica personal que se va edificando atreves del crecimiento psicológico de la persona y de la capacidad para deliberar y de relacionarse de forma adecuada con las experiencias de la vida, el bienestar además depende de algunas variables como edad, género y cultura que deben de ser analizados en varios contextos [8].

Luego que la Organización Mundial de la Salud (OMS), a inicios de marzo del año 2020, declaró al COVID - 19 como una pandemia [9], es indispensable señalar que el impacto a nivel psicológico es más relevante en personas que viven la situación en primera línea [6], como son los profesionales de la salud y los estudiantes de las carreras afines que realizan las practicas pre - profesionales, razón en la que radica la importancia de este estudio, además que es relevante que ante una emergencia sanitaria el personal de salud tome medidas para garantizar su salud mental debido a que las condiciones laborales ante este tipo de situación exigen un alto esfuerzo psicológico, mental, emocional y conductual [10].

En tal sentido, de acuerdo con el contexto y la situación actual de esta población el siguiente trabajo muestra los resultados de un estudio cuyo objetivo es determinar el impacto psicológico frente al COVID - 19 de los internos de enfermería de las practicas pre - profesionales, tomando en cuenta las características sociodemográficas, el área de rotación y las relaciones entre la ansiedad, depresión, malestar psicológico y el miedo frente a la enfermedad.

\section{Desarrollo}

El bienestar psicológico se ha enmarcado a lo largo de numerosos estudios como parte de la ecuanimidad del individuo, que esta expresada por autopercepciones emocionales, y por habilidades como la regulación emocional y emulación social [11], y han sido considerados como el equivalente de felicidad, calidad de vida, salud mental, resiliencia y motivación [12].

Estudios realizados durante la pandemia [13], [14], [15] han demostrado que los profesionales de la salud han pedido a sus organizaciones cinco cosas indispensables que son: cuídame, escúchame, protégeme, prepárame y apóyame; por lo tanto, es indispensable la creación de un ambiente psicológico seguro, liderazgo firme, estrategias organizativas concisas, apoyo y comunicación constante con todo el equipo para fomentar una cultura de resiliencia que previenen manifestaciones psicológicas negativas en el contexto de la pandemia por COVID-19, por otra parte la situación actual exige el afrontamiento de situaciones entre las q se encuentra el desbordamiento del sistema de salud, el riesgo de contagio, la falta de recursos e insumos para su protección, el miedo a llevar la infección a sus familiares, estrés en zonas de atención directa por abrumadora carga de trabajo y dilemas éticos y morales [16].

De acuerdo con información ofrecida por la Sociedad Española de Psiquiatría (SEP), la salud mental del personal sanitario se ve modificada por los retos a los que se enfrentan en la atención a pacientes y familiares [17]. Las recomendaciones realizadas por la SEP, demandan mecanismos de afrontamiento para satisfacer de forma efectiva las necesidades básicas como descanso, relaciones adecuadas con los compañeros de labores, compartir información constructiva, estar en contacto con la familia, restringir la exposición al riesgo, controlar la recepción de información y el uso de los medios de comunicación, autobservación para ser consciente de sus emociones, aplicación de técnicas de regulación emocional y recordar que lo que es posible no siempre es probable [17].

En pandemias anteriores se observó que los trabajadores de salud además de sufrir afectación directa en su bienestar psicológico, presentaron resultados negativos en evaluaciones psicológicas un año después de estos eventos lo que se traduce en estrés postraumático [13], que incluye un cuadro de conductas de evitación, altos niveles de ansiedad y preocupación ante el hecho de la posible infección, las cuales repercuten en la vida cotidiana de quienes enfrentan esta situación.

Respecto a la pandemia del COVID-19, un estudio realizado en China indica que el nivel psicológico del personal de salud se ve mayormente afectado en mujeres, involucrando en superior cuantía a las enfermeras que a los médicos [18]. Con relación a las consecuencias que se manifiestan en el personal de salud, que incluye a los estudiantes de las carreras afines que realizan sus prácticas pre-profesionales, están los efectos a corto plazo que abarcan la pérdida de apetito, irritabilidad e insomnio; y a largo plazo están los trastornos músculo esquelético, del sueño, y alteraciones cardiovasculares y gastrointestinales [19].Resultados de encuestas realizadas en 
varias ciudades chinas informan que el impacto psicológico se determinó de moderado a severo, el que afecta mayoritariamente a la población que palpa de cerca esta enfermedad como son el personal de salud y los estudiantes que cursan su prácticas pre - profesionales [20].

\section{Metodología}

Se incluyeron 150 internos prácticos de la carrera de Enfermería, por muestreo aleatorio simple de acuerdo a la fórmula de Sierra Bravo de 1988[21], el error (5\%) que cometemos de estimación del tamaño de la muestra, partiendo un nivel de confianza del 95\% seguiría la siguiente formula, tomando $\mathrm{Z}=1,96$

$$
n=\frac{Z_{\forall}^{2} * p * q * N}{e^{2}(N-1)+Z_{\forall}^{2} p * q}
$$

Los instrumentos utilizados fueron una encuesta con variables Sociodemográficas: Edad, género, estado civil y área de rotación. El Cuestionario de ansiedad Estado - Rango (STAI): compuesta por 40 ítems dividido en dos sub-escalas en un método de respuesta Likert de 4 opciones según la intensidad [22].

Inventario de depresión estado - rango (IDER): Un instrumento corto de 20 ítems, que identifica del grado de afectación (estado) y la frecuencia de la ocurrencia (rango) del componente afectivo de la depresión; se obtienen 3 puntua- ciones de eutumia, distimia y la puntuación total del estado y rasgo medidas con el método de repuesta Likert [23].

FCV19S: Escala de 7 ítems, con auto informe del miedo emocional específicamente al COVID - 19 [24].

Malestar Psicológico (K10): de 10 ítems, con 5 opciones de respuesta [25].

Para la aplicación del instrumento inicialmente, se solicitó la autorización a las autoridades de la Universidad, luego el instrumento fue adaptado al formato Google forms y enviado a los correos electrónicos de los estudiantes previa información del objetivo de la investigación, las instrucciones de la escala y la confidencialidad de los datos proporcionados, aceptando participar voluntariamente firmando el consentimiento informado respectivo.

\section{Resultados}

Para el procedimiento se realizó un análisis descriptivo de las características sociodemográficas, la ansiedad, la depresión, el miedo al COVID-19 y el malestar psicológico en la población de estudio mediante frecuencias, porcentajes (variables cualitativas) y medidas de tendencia central (variables cuantitativas). Se utilizó pruebas paramétricas, para analizar si la ansiedad, la depresión y el malestar psicológico se relacionan con el miedo al COVID-19, y también la $\mathrm{r}$ de Pearson y para verificar la ansiedad, depresión, malestar psicológico y miedo de acuerdo al área de rotación, Los análisis estadísticos se realizarán mediante el programa estadísticos infostat y SPSS 26.

Tabla 1. Características sociodemográficas.

\begin{tabular}{|c|c|c|c|}
\hline & & $\begin{array}{c}f \\
\mathrm{n}=150\end{array}$ & $\%$ \\
\hline \multirow{2}{*}{ Sexo } & Mujer & 127 & 84.7 \\
\hline & Varón & 23 & 15.3 \\
\hline \multirow{2}{*}{ Provincia } & Azuay & 68 & 45.3 \\
\hline & Cañar & 82 & 54.7 \\
\hline \multirow{5}{*}{ Estado civil } & Casado & 13 & 8.7 \\
\hline & Divorcia & 2 & 1.3 \\
\hline & Separado & 2 & 1.3 \\
\hline & Soltero & 116 & 77.3 \\
\hline & Unión Libre & 17 & 11.3 \\
\hline \multirow{2}{*}{ Área de rotación } & Comunitaria & 57 & 38.0 \\
\hline & Hospital & 93 & 62.0 \\
\hline \multirow{2}{*}{ Cuenta con equipos de bioseguridad } & No & 21 & 14.0 \\
\hline & $\mathrm{Si}$ & 129 & 86.0 \\
\hline \multirow{5}{*}{ Qué sentimiento le genera la pandemia } & Ansiedad & 52 & 34.7 \\
\hline & Enojo & 12 & 8.0 \\
\hline & Me es indiferente & 17 & 11.3 \\
\hline & Miedo & 67 & 44.7 \\
\hline & Pánico & 2 & 1.3 \\
\hline \multirow{3}{*}{ Padece alguna enfermedad } & $\begin{array}{l}\text { Enfermedades } \\
\text { respiratorias }\end{array}$ & 1 & .7 \\
\hline & Hipertensión & 2 & 1.3 \\
\hline & Ninguna & 147 & 98.0 \\
\hline
\end{tabular}


La tabla 1 describe las características sociodemográficas incluyendo el área de rotación, la cual indica que 57 internos pertenecen al área comunitaria (38\%) y 93 al área hospitalaria $(62 \%)$, lo cual puede representar un aspecto importante para las manifestaciones de miedo o ansiedad, ya que las áreas comunitarias manejan pacientes ambulatorios, mientras que las áreas hospitalarias están destinados a la atención directa de pacientes diagnosticados como positivos para la enfermedad.

En cuanto a los equipos de seguridad el $86 \%$ de internos declararon que, si cuentan con los equipos, es importante mencionar este dato ya que el $62.7 \%$ de internos estuvieron en contacto con pacientes COVID-19. Los equipos de seguridad más utilizados son mascarillas, batas y gorros descartables y de esta forma permiten mayor movilidad de los internos, y al mismo tiempo ofrecen una protección apropiada disminuyendo los niveles de ansiedad, depresión y miedo.

A.Niveles de ansiedad en los internos de enfermería de prácticas pre-profesionales por el COVID-19

Para medir la ansiedad de la muestra, en esta investigación se utilizó el Cuestionario de Ansiedad Estado Rango (STAI) [22] compuesto por dos escalas de autoevaluación para medir los conceptos independientes de la ansiedad. La ansiedad estado (AE) la definen los autores como una condición emocional transitoria, caracterizada por sentimientos de tensión y aprensión, mientras que la ansiedad rasgo (AR) la definen como una propensión ansiosa estable donde las personas y situaciones se perciben como amenazadoras, aumentando la ansiedad [26].

La corrección se realiza con una plantilla en la cual se cuenta los ítems de ansiedad positivos y negativos, para estado (AE+, $\mathrm{AE}-$ ) y para rango (AR+, $\mathrm{AR}-$ ).

Para obtener la suma correspondiente a cada sub-escala, debe aplicarse a las ecuaciones (2) y (3):

$$
\begin{aligned}
& A E=30+(A E+)-(A E-) \\
& A R=21+(A R+)-(A R-)
\end{aligned}
$$

En base a los resultados obtenidos en las ecuaciones (2) y (3) es posible caracterizar el estado de ansiedad de los encuestados tomando en cuenta los rangos presentados en la Tabla 2.

Tabla 2. Rangos para el estado de ansiedad.

\begin{tabular}{cc}
\hline Ansiedad & Rango \\
Normal & $0-19$ \\
Leve & $20-28$ \\
Alta & $29-39$ \\
Severa & $>40$ \\
\hline
\end{tabular}

Con valores de ansiedad estado AE y rango (AR) mayoritariamente entre 22 y 28 , los internos presentan una escala de ansiedad leve. En cuanto al sexo las mujeres presentan medias de AE y AR más alto que los hombres con diferencias de (3.19 y 1.38 respectivamente). Los factores asociados con un alto niveles de ansiedad estado y rango fueron aquellos internos que declararon no contar con los equipos de bioseguridad. Con una media de 40.14 para el AE y 33.33 para el AR indican un nivel severo de ansiedad. Resultados similares se evidencia en el estudio [27] en el que se dedujo que la usencia del equipo de protección básico como son las mascarillas N95, mascarillas quirúrgicas, batas descartables, guantes descartables y visores; genera diferentes grados de ansiedad, encontrándose que el $83 \%$ de los profesionales dieron niveles altos de ansiedad. Además, se dedujo un nivel de seguridad calificado como medio para aquellos que usan los equipos de protección en su jornada laboral.

La media de los valores de la escala IDER [23], están en rangos de por debajo de los percentiles 75; por lo tanto, se puede determinar que los internos de enfermería de las practicas pre-profesionales no presentan depresión, pues valore menores al percentil 75, equivalente a 23 en la escala IDER, indican valores normales. Importante mencionar que en esta categoría destaca un factor correspondiente a aquellos que declararon tener enfermedades como son las enfermedades respiratorias e hipertensión, pues la escala IDER es está entre 25-28, es decir que estos internos son susceptibles a desarrollar estados depresivos. No hubo diferencia significativa en mujeres y varones.

B.Niveles de miedo en los internos de enfermería de las prácticas pre-profesionales por el COVID-19

Se aplicó el cuestionario Fear COVID-19 Scale (FCV19S) [24], y se determinó que la muestra en general posee un miedo alto frente al COVID-19. Presentaron mayores niveles de miedo aquellos internos que se presentan en la etapa inicial de la rotación $(\mathrm{FCV}-19 \mathrm{~S}=25)$ debido a la poca experiencia que poseen y aquellos internos que tienen hipertensión $(\mathrm{FCV}-19 \mathrm{~S}=26)$ atribuyéndose a las posibles complicaciones en caso de contagiarse de la enfermedad. Sin embargo, tanto las mujeres como los varones mostraron niveles similares de miedo (21.0 y 20.4 respectivamente).

C.Niveles de malestar psicológico en los internos de enfermería de prácticas pre-profesionales por el COVID-19

Por último, dentro de este apartado se evaluó el malestar psicológico mediante la aplicación del cuestionario K-10 de Kessler [25]. Se puedo establecer que el $100 \%$ de la población presentaron niveles altos de malestar psicológico, ya que los valores de la escala K-10 predominan para el rango de 22-30 [25]. Con malestar psicológico muy alto se encuentran aquellos internos que padecen de enfermedades respiratorias, con un valor en la escala de 32.00 , pero este factor corresponde tan sólo al $0.7 \%$ de la población. Es importante mencionar este hecho, ya que este puede ser considerado una línea base para futuras investigaciones orientado a evaluar el impacto psicológico en personas con enfermedades respiratorias y/o especiales. No hubo diferencia significativa de malestar psicológico entre mujeres y varones. 
Tabla 3. Índices de correlación entre la ansiedad, depresión, miedo y malestar psicológico.

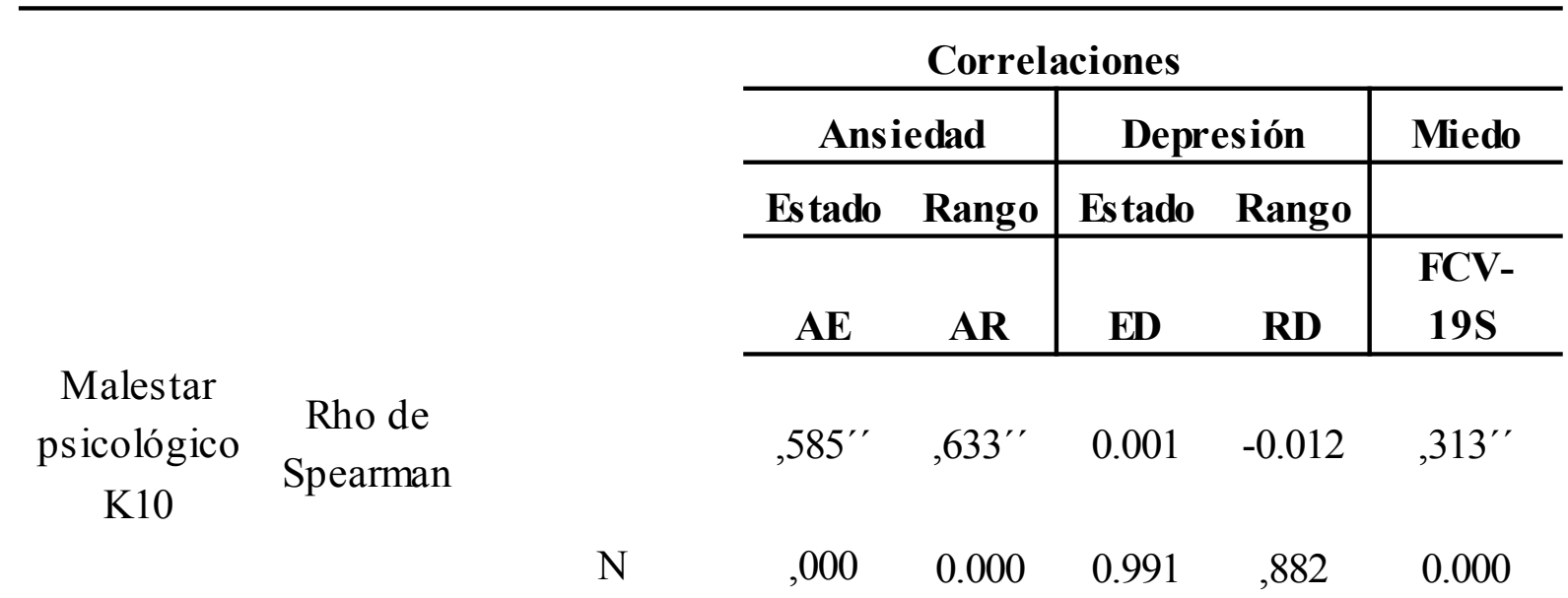

Nota: Valor de $p$ con negrita.

**. La correlación es significativa al nivel 0.01

*. La correlación es significativa al nivel 0.05

Las correlaciones halladas en ansiedad estado y rango fueron moderadas y altamente significativas, con coeficientes de correlación que oscilan entre los índices 0.585 y 0.622 . Las correlaciones halladas entre la depresión estado y rango con el malestar psicológico fueron bajas, con valores cercanos a cero. La correlación de la escala miedo con el malestar psicológico fue media y altamente significativas con un coeficiente de correlación de 0.313 .

Los valores de escala demuestran que los internos del área hospitalaria presentan mayores niveles de ansiedad, miedo y malestar psicológico compara con los internos del área comunitaria con pequeñas diferencias (diferencia ansiedad $\mathrm{AE}=2.37, \mathrm{AR}=3.07$; diferencia miedo $\mathrm{FCV}-19 \mathrm{~S}=0.5$; diferencia malestar $\mathrm{K} 10=0.23$ ). Difiere en cuanto a depresión, ya que los niveles de depresión son mayores para los internos que rotan en el área comunitaria (diferencia depresión $\mathrm{DE}=$ $0.3, \mathrm{DR}=0.9$ ).

\section{Conclusiones}

Los resultados obtenidos en el presente trabajo evidencian que los internos de enfermería que realizan las practicas pre-profesionales presentan niveles de ansiedad, miedo y malestar psicológico altos como consecuencia de la pandemia del COVID-19. Por ello es muy importante cuidar a los profesionales que están en primera línea durante esta pandemia del COVID-19. Por lo contrario, no presentaron tener niveles altos de depresión.

No hubo diferencia significativa de los estados emocionales entre mujeres y varones.

Se evidenció en los niveles de ansiedad severos de los internos que declararon no tener los equipos protectores de bioseguridad.

Existe una correlación de moderada a alta y significativa entre la ansiedad, miedo y malestar psicológico.

Los internos que rotan en el área hospitalaria presentan mayores niveles de ansiedad, miedo y malestar psicológico compara con los internos del área comunitaria. Sin embargo, los niveles de depresión fueron ligeramente mayores en los internos de área comunitaria.

Los hallazgos del estudio pueden usarse para formular intervenciones psicológicas para mejorar la salud mental de las poblaciones vulnerables durante la epidemia del COVID- 19.

\section{Agradecimiento}

A la Coordinadora y Docentes de la Maestría en Gestión del Cuidado de la Universidad Católica de Cuenca y al Laboratorio de Psicometría del Centro de Investigación, Innovación y Transferencia de Tecnología (CIITT).

\section{Fuente de Financiamiento}

Este estudio es autofinanciado

\section{Conflicto de Interés}

No existen conflictos personales, profesionales o de otro tipo.

\section{Referencias}

[1]M. Del Campo, «Covid-19 y su impacto en la salud y el trabajo,» Revista de la Asociación Española de Especialistas en Medicina del Trabajo, vol. 29, nº 3, p. 183, 2020. 
[2]H. Scholten, VE. Quezada-Scholz, G.Salas, NA. Barria-Aseenio, R. Molina, JE. García, M. Juliá, A. Marinero, A. Zambrano, E. Gómez, A. Cheroni, T. Caycho-Rodriguez, T. Reyes, N. Pinochet, P. Binde, J. Uribe, C. Rojas, J. Bernal, F. Somarriva, "Abordaje psicológico del COVID-19: Una revisión narrativa de la experiencia latinoamericana," Rev Interam Psicol J Psychol, vol. 54, pp.287, May. 2020.

[3]A. Tusev, L. Tonon, y M. Capella, "Efectos Iniciales en la Salud Mental por la Pandemia de Covid-19 en algunas Provincias de Ecuador", IRR, vol. 15, pp. 11-24, oct. 2020.

[4]Ministerio de Salud Pública (2020, marzo 13) Informe de situación $\mathrm{N}^{\circ}$ 001.[Online]. Disponible en: https:/www. gestionderiesgos.gob.ec/wp-content/uploads/2020/03/Informe-de-Situaci\%C3\%B3n-No001-Casos-Coronavirus-Ecuador-12032020.pdf

[5]F. De Caneva, M. García-Gómez, E Baur, "Salud emocional del personal sanitario del Área de Urgencias durante la pandemia COVID-19”, Rev. Med Clin. Vol. 155, no. 8, pp. 365 , junio 2020.

[6]S Dubey, P. Biswas, R. Ghosh, S. Chatterjee, MJ. Dubey, S. Chatterjee, D. Lahiri, CJ. Lavie, "Psychosocial impact of COVID-19”, Rev Diabetes Metab Syndr. Vol.14, no. 5, pp. 779-788, septiembre-octubre. 2020.

[7]G. Arias, F. Navarrete, M. Flor, "Factores asociados al bienestar psicológico de los estudiantes de la escuela académico-profesional de odontología de la Universidad nacional Jorge Basadre Grohmann", Revista médica Basadrina. Vol. 7, no. 2, pp. 28-30, septiembre. 2013.

[8]S. Sandoval, A. Dorner, A. Véliz, "Bienestar psicológico en estudiantes de carreras de la salud," Rev Investigación en Educación Médica, Vol. 6, no. 24, pp.260-266, diciembre 2017.

[9]C. Mitchell, OPS/OMS | La OMS (2020, octubre 23) caracteriza a COVID-19 como una pandemia. Pan American Health Organization / World Health Organization [online] Disponible en: https:/www.paho.org/hq/index.php?option=com_content\&view $=$ article \&id=15756: who-characterizes-covid-19-as-a-pandemic\&Itemid $=1926 \&$ lang $=\mathrm{es}$

[10]MC. Johnson, L. Saletti-Cuesta, N. Tumas, "Emociones, preocupaciones y reflexiones frente a la pandemia del COVID-19 en Argentina," Rev Ciênc Saúde Coletiva, vol 25(suppl 1), pp. 2447-56, junio. 2020.

[11]L. Hernani, A. Aquino, E. Araujo, "Relación entre el bienestar auto percibido, autoestima, inteligencia emocional, personalidad y razonamiento, abstracto en un grupo de estudiantes universitarios," Revista peruana de Psicología y trabajo social, vol. 2, no. 1, pp. 49-62, mayo. 2013.

[12]N. Ozamiz-Etxebarria, M. Dosil-Santamaria, M. Picaza-Gorrochategui, N. Idoiaga-Mondragon, "Niveles de estrés, ansiedad y depresión en la primera fase del brote del COVID-19 en una muestra recogida en el norte de España," Cad Saúde Pública, vol. 36, no. 4, abril. 2020.

[13]H. Blake, F. Bermingham, G. Johnson, and A. Tabner, "Mitigating the Psychological Impact of COVID-19 on Healthcare Workers: A Digital Learning Package," International Journal of Environmental Research and Public Health, vol.
17, no. 9, pp. 2997, abril 2020.

[14]M. Walton, E. Murray, MD. Christian, "Mental health care for medical staff and affiliated healthcare workers during the COVID-19 pandemic," Rev. Eur Heart J Acute Cardiovasc Care, vol. 9, no. 3, pp. 241-247, Abril. 2020.

[15]J. Shijo, P. Gunaseelan, S. Bhandari, S. Dutta, "How the novel coronavirus (COVID-19) could have a quivering impact on mental health?," Rev. Open J Psychiatry Allied Sci, vol.11, no. 2, pp.135-136, julio-diciembre. 2020.

[16]W. El-Hage, C. Hingray, C. Lemogne, A. Yrondi, P. Brunault, T. Bienvenu, B. Etain, C. Paquet, B. Gohier, D Bennabi, P. Birmes, A. Sauvaget, E. Fakra, N. Prieto, S. Bulteau, P. Vidailhet, V. Camus, M. Levoyer, M. Krebs, B. Aouizerate, "Les professionnels de santé face à la pandémie de la maladie à coronavirus (COVID-19): quels risques pour leur santé mentale?," Rev. L'Encephale, vol. 46, no. 3, pp.73-80, octubre, 2020 .

[17]Sociedad Española de psiquiatría. "Cuidado de la salud mental del personal sanitario". 2020 Disponible en: http:// www.sepsiq.org/file/InformacionSM/SEP\%20COVID19-Salud\%20Mental\%20personal\%20sanitario.pdf

[18]A. Lozano-Vargas, "Impacto de la epidemia del Coronavirus (COVID-19) en la salud mental del personal de salud y en la población general de China," Rev Neuropsiquiatr, vol. 83, pp. 51-6, enero, 2020.

[19]D. Talevi, V. Socci, M. Carai, G. Carnaghi, S. Faleri, E. Trebbi, A. Di Bernardo, F. Capelli, F. Pacitti, "Mental health outcomes of the CoViD-19 pandemic," Riv Psichiatr, vol. 55, no. 3, pp. 137-144, mayo-junio, 2020.

[20]A. Urzúa, P. Vera-Villarroel, A. Caqueo-Urízar, R. Polanco-Carrasco, "La Psicología en la prevención y manejo del COVID-19. Aportes desde la evidencia inicial," Rev. Ter Psicológica, vol. 38, pp. 103-18, abril, 2020.

[21]R. Sierra. Técnicas de investigación social. 9na Edición. Madrid. Ed.Paraninfo S.A. 1988.

[22]C. Spielberger, R. Gorsuch, R. Lushene. "Cuestionario de Ansiedad Estado-Rasgo". TEA: Ediciones, 2015. Disponible en: http://www.web.teaediciones.com/Ejemplos/ STAI-Manual-Extracto.pdf

[23]D. Gudelo, Y. Gómez, P. López, “Propiedades Psicométricas del Inventario de Depresión Estado Rasgo (IDER) con una muestra de población general colombiana," Rev. Av En Psicol Latinoam, vol. 32, no 1, pp. 71-84, 2014.

[24]D. Tzur, A. Grossman-Giron, Y. Bloch, Y. Mayer, N. Shiffman, S. Mendlovic, "Fear of COVID-19 scale: Psychometric characteristics, reliability and validity in the Israeli population," Rev.Psychiatry, vol. 289, pp. 113100, julio, 2020. [25]B. Vargas, V. Villamil, C. Rodríguez, J. Pérez, S. Cortés, "Validación de la escala Kessler 10 (K-10) en la detección de depresión y ansiedad en el primer nivel de atención. Propiedades psicométricas," Rev. Salud Ment, vol. 34, pp. 323-331, julio-agosto 2011. 


\section{RESUMEN CURRICULAR}

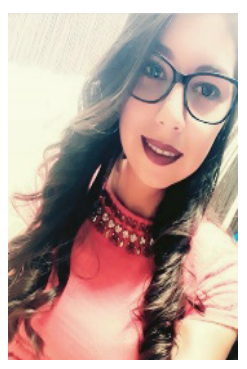

María José Mera Navarrete, Licenciada en Enfermería, Estudiante de la Maestría en Gestión del Cuidado, Universidad Católica de Cuenca. Docente tutor de prácticas pre profesionales en la Universidad Católica de Cuenca.

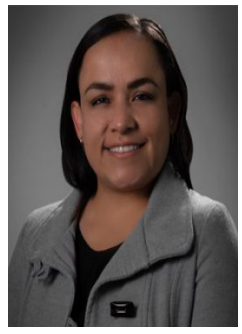

Isabel Cristina Mesa Cano, Enfermera especialista en Cuidado al Adulto en Estado Crítico de Salud, Doctorado en Enfermería por la Universidad Andrés Bello, Chile. Coordinadora de la Maestría en Gestión del Cuidado. Docente de la Universidad Católica de Cuenca

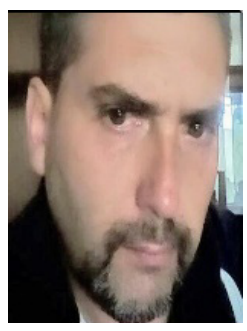

Pedro Carlos Martínez Suarez, Doctor, PhD en Psicología por la Universidad de Oviedo, España; Vicerrector de investigación e innovación, vinculación y posgrado de la Universidad Católica de Cuenca.

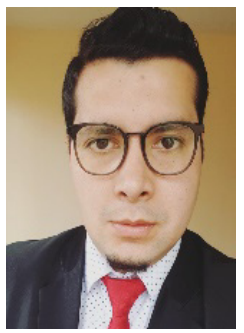

Andrés Alexis Ramírez Coronel, psicólogo, Máster en neuropsicología, Doctorado en epidemiologia y bioestadística. Docente de la Maestría en Gestión del Cuidado. 